\title{
Review Article \\ Similarities and Differences between the Pathogenesis and Pathophysiology of Diastolic and Systolic Heart Failure
}

\author{
Kazuo Komamura \\ Cardiovascular Division, Hyogo College of Medicine, 1-1 Mukogawa-cho, Nishinomiya, Hyogo 663-8501, Japan \\ Correspondence should be addressed to Kazuo Komamura; komamura@hyo-med.ac.jp
}

Received 19 August 2013; Revised 8 October 2013; Accepted 10 October 2013

Academic Editor: Dirk Westermann

Copyright (C) 2013 Kazuo Komamura. This is an open access article distributed under the Creative Commons Attribution License, which permits unrestricted use, distribution, and reproduction in any medium, provided the original work is properly cited.

\begin{abstract}
Pathophysiology of heart failure has been considered to be a damaged state of systolic function of the heart followed by a state of low cardiac output that is, systolic heart failure. Even if systolic function is preserved, left ventricular filling in diastole can be impeded and resulted in elevation of filling pressure and symptoms of heart failure. This kind of heart failure is called diastolic heart failure. Nowadays, diastolic heart failure is referred to as heart failure with preserved ejection fraction (HFpEF), whereas systolic heart failure is referred to as heart failure with reduced ejection fraction (HFrEF). In this paper, the similarities and differences between the pathogenesis and pathophysiology of diastolic and systolic heart failure were reviewed. Although diastolic heart failure is a common condition of heart failure worldwide, its pathophysiology has not been sufficiently elucidated. This is thought to be the most significant reason for a lack of established treatment methods for diastolic heart failure. We hope to proceed with future studies on this topic.
\end{abstract}

\section{Introduction}

Pathophysiology of heart failure has been considered to be a damaged state of systolic function of the heart followed by a state of low cardiac output (systolic heart failure). However, even if systolic function is preserved, left ventricular filling in diastole is impeded due to various factors. This condition leads to congestive heart failure due to the rise in left ventricular end-diastolic pressure and the decrease in cardiac output. This kind of pathophysiology is now known as diastolic heart failure $[1,2]$. In recent years, diastolic heart failure caused by the affected left ventricle has become a clinical issue [3]. Nowadays, diastolic heart failure is referred to as heart failure with preserved ejection fraction (HFpEF), whereas systolic heart failure is referred to as heart failure with reduced ejection fraction (HFrEF). This is because evaluating accurate pathophysiology and diagnosis of diastolic heart failure is in fact difficult.

\section{Diastolic Dysfunction}

Diastole of the left ventricle is composed of isovolumic relaxation and ventricular filling. Relaxation of the left ventricle is an active process that occurs as a result of energy-dependent uptake of intracellular calcium by the sarcoplasmic reticulum, whose concentration has risen during the systolic phase.

Relaxation of the left ventricle is impaired in a disease state caused by energy metabolism disorders or calciumhandling abnormalities such as myocardial ischemia and myocardial hypertrophy. Left ventricular filling phase abnormality, namely, elevation of left ventricular stiffness, influences left ventricular flow dynamics during filling phase and is commonly caused by myocardial fibrosis or hypertrophy.

When left ventricular diastolic function is impaired, cardiac output is reduced because the left ventricle is not filled enough in diastole due to left ventricular inflow obstruction. By contrast, to compensate for reduced cardiac output, increasing the inflow pressure to the left ventricle (and consequently left ventricular end-diastolic pressure) becomes necessary, which in turn increases left atrial pressure. As a result, left ventricular dysfunction easily and directly causes pulmonary congestion.

The end-systolic pressure-volume relationship is the same as a normal heart in diastolic heart failure, but the end-diastolic pressure-volume relationship shifts upwards (Figure 1(a)) [3]. As a result, left ventricular end-diastolic 


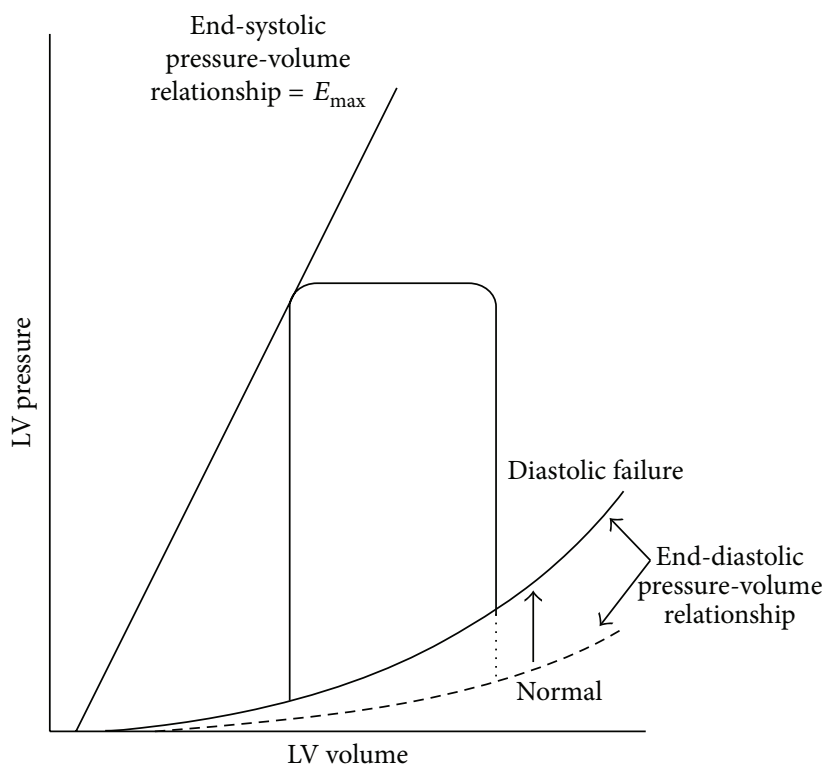

(a)

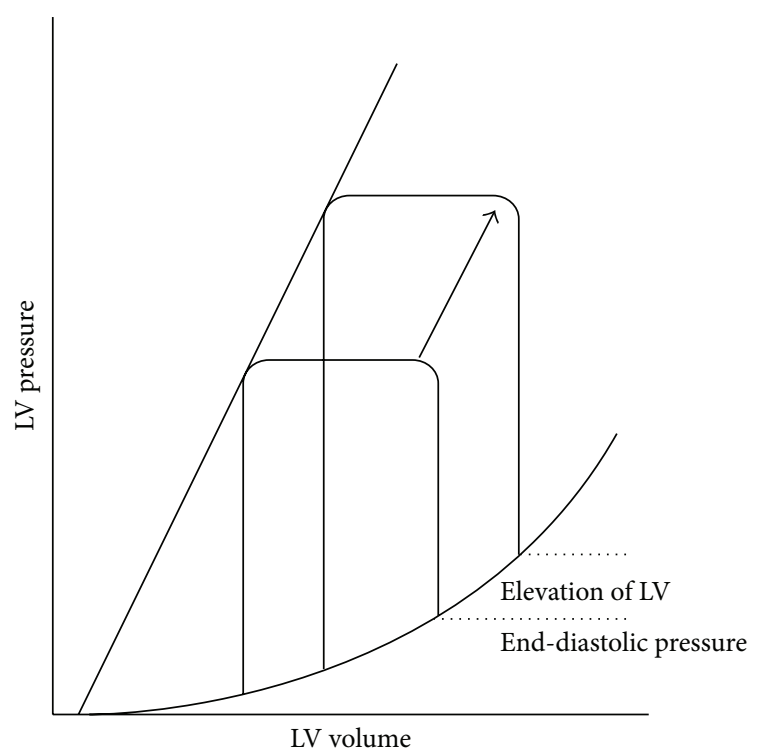

(b)

Figure 1: (a) The end-systolic pressure-volume relationship is the same as a normal heart in diastolic heart failure, but the end-diastolic pressure-volume relationship shifts upwards. As a result, left ventricular end-diastolic pressure rises. (b) In pathologies with diastolic dysfunction, when an abrupt increase in blood pressure occurs, the pressure-volume loop shifts to the upper right without decrease in $E_{\max }$. Therefore, pulmonary congestion is induced as a result of the significant increase in left ventricular end-diastolic pressure. LV: left ventricular.

pressure rises. In pathologies with diastolic dysfunction, when an abrupt increase in blood pressure occurs, the pressure-volume loop shifts to the upper right without decrease in $E_{\max }$ (absolute index of contractibility). Therefore, pulmonary congestion is induced as a result of the significant increase in left ventricular end-diastolic pressure (Figure 1(b)).

By contrast, in systolic dysfunction, left ventricular contractile function decreases and $E_{\max }$ gets smaller (Figure 2). Meanwhile, the end-diastolic pressure-volume relationship shifts downwards rather than remaining unchanged. To maintain cardiac output, the pressure-volume loop shifts right due to increase in preload. Therefore, the left ventricular pressure-volume loop operates on the steep part of the enddiastolic pressure-volume curve, consequently causing enddiastolic pressure to rise.

\section{Are Diastolic Dysfunction and Systolic Dysfunction Separate Diseases?}

In diastolic dysfunction, contractility of whole left ventricle is considered normal. However, the contractile velocity in systole measured with tissue Doppler decreased in both systolic and diastolic dysfunction [4]. Furthermore, local contractility in longitudinal direction is known to be impaired locally in diastolic heart failure [5]. Recent findings suggest that contractility decreases even in diastolic heart failure in myocardium level. By contrast, diastolic function is also impaired in systolic heart failure and has been shown to decrease exercise tolerance and be one of the determinants of prognosis [6]. Therefore, diastolic and systolic heart failure are not considered to be independent and separate entities. The single syndrome hypothesis of heart failure is therefore advocated (Figure 3) [1]. In that hypothesis, heart failure is a single continuous disease spectrum and systolic and diastolic heart failure are phenotypes at two extremes.

Thus, there is the "grey zone" in diagnosing HFrEF with LVEF of 45 to $50 \%$ or 45 to $55 \%$. In other words, some of HFpEF with LVEF of 45 to $55 \%$ might be diagnosed as HFrEF rather than HFpEF. As shown in Figure 3, a phenotype of heart failure comprised of some extent of systolic dysfunction and some extent of diastolic function. Heart failure with LVEF of 45 to $55 \%$ would be located in the middle of the continuum of disease spectrum.

By contrast, some researchers have advocated that diastolic function is not something that should only be noted in the pathogenesis of diastolic heart failure, but should be widely viewed as a determinant of pathophysiology in heart failure [7]. Heart dysfunction that occurs as a result of heart disease causes diastolic dysfunction. Among such cases there exist patients with concurrent systolic dysfunction. Furthermore, a portion of patients with heart dysfunction clinically exhibit symptoms of heart failure. Among them, those with significant systolic dysfunction where the main pathology is systolic heart failure, and diastolic dysfunction, are said to have diastolic heart failure.

\section{The Diagnostic Criteria for Diastolic Heart Failure}

Definition of systolic heart failure is reduction of the left ventricular ejection fraction. Thus, its diagnosis is quite easy. By contrast, diagnosis of diastolic heart failure is difficult 


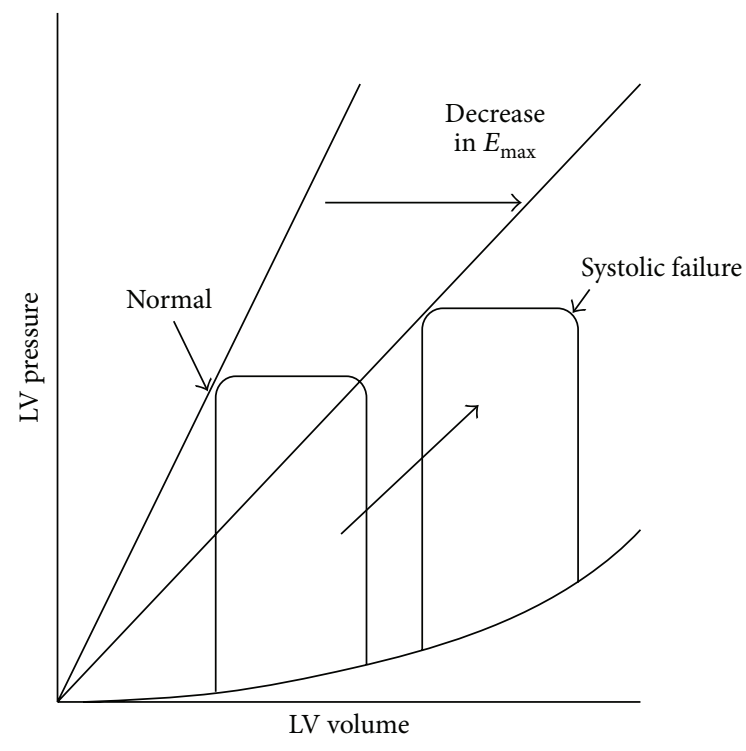

FIGURE 2: In systolic dysfunction, LV contractile function decreases and $E_{\max }$ gets smaller. Meanwhile, the end-diastolic pressurevolume relationship shifts downwards rather than remaining unchanged. To maintain cardiac output, the pressure-volume loop shifts right due to increase in preload. Therefore, the LV pressurevolume loop operates on the steep part of the end-diastolic pressurevolume curve, consequently causing end-diastolic pressure to rise. $\mathrm{LV}$ : left ventricular.

Diastolic heart failure

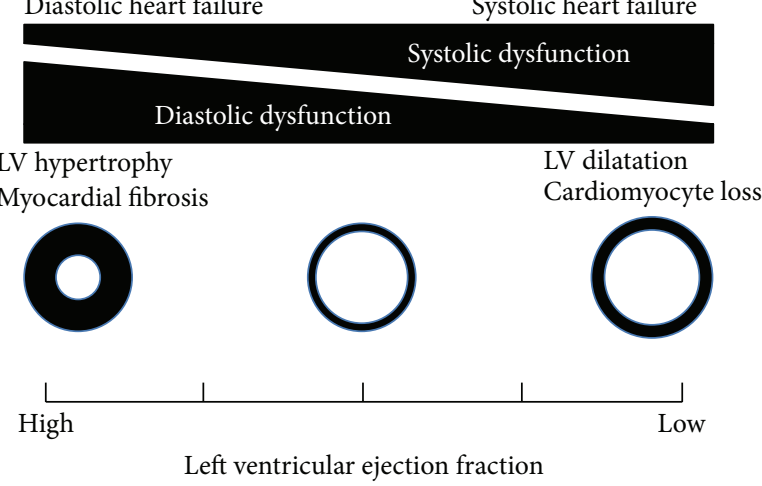

Figure 3: Diastolic and systolic heart failure are not considered to be independent and separate entities. Heart failure is a single continuous disease spectrum and systolic and diastolic heart failure are phenotypes at two extremes.

since there are no simple and reliable criteria. Therefore, diastolic heart failure can be clinically diagnosed when clinical symptoms and findings of heart failure are exhibited and decrease in left ventricular ejection fraction is none or minimal.

The American College of Cardiology Foundation and the American Heart Association define diastolic heart failure as a condition having the typical signs and symptoms of heart failure with a normal left ventricular ejection fraction, without valvular abnormalities on echocardiography [8]. Vasan and Levy define diastolic heart failure as (1) exhibiting clinical symptoms of congestive heart failure, (2) having normal left ventricular systolic function during congestive heart failure (left ventricular ejection fraction of 45 to $50 \%$ and above), and (3) having left ventricular diastolic dysfunction. Those that meet all these items and who have congestive heart failure that is not caused by valvular heart disease, cor pulmonale, or primary volume overload are considered as "definite diastolic heart failure (definite DHF)" cases. Currently, accurate diagnosis of (3) needs cardiac catheterization, which could be skipped in a common clinical situation.

Diastolic heart failure is strongly suspected (probable DHF) when conditions (1) and (2) are met [9]. When diagnosing diastolic heart failure, it is important to perform a careful exclusion of valvular heart disease, pericardial disease, right heart failure, intracardiac tumor, congenital heart disease, and high-output cardiac failure. Zile et al. demonstrated that diastolic functional abnormalities caused an increase in left ventricular filling pressure and clinical symptoms of congestive heart failure using cardiac catheterization for patients with heart failure with preserved ejection fraction and patients with diastolic heart failure $[10,11]$. The currently accepted criteria for diagnosis of diastolic heart failure are essentially a clinical diagnosis. Thus, it is important to understand that there are possibilities that diastolic heart failure means somewhat broader range than what diastolic heart failure exactly stands for.

\section{Diastolic Heart Failure from the Perspective of Clinical Features}

According to reports by Owan et al. and Bhatia et al., roughly half of hospitalizations for heart failure are due to diastolic heart failure $[12,13]$. Compared with systolic heart failure, diastolic heart failure is seen more often in the elderly and women and accompanied by hypertension and anemia. Comorbidity rate of obesity, diabetes, and chronic kidney disease (CKD) in diastolic heart failure is high, but not particularly higher than in systolic heart disease. In general, both diastolic and systolic heart failure exhibit distinctive subjective symptoms and objective findings of heart failure including dyspnea, edema, and malaise. Symptoms of diastolic heart failure typically include dyspnea due to pulmonary congestion, particularly shortness of breath, paroxysmal atrial fibrillation, and rapidly developing dyspnea induced by tachycardia, all of which are common initial symptoms. By contrast, in systolic heart failure, symptoms and signs due to general malaise and organ hypoperfusion associated with decreased cardiac output are frequently seen.

The main differences between diastolic and systolic heart failure are the presence of contractile dysfunction and left ventricular remodeling (Table 1). In systolic heart failure, progressive ventricular dilatation, or eccentric cardiac hypertrophy, can be seen. By contrast, diastolic heart failure exhibits concentric ventricular remodeling without dilatation or concentric cardiac hypertrophy. The tissue Doppler E/E' ratio (early mitral inflow peak velocity/early diastolic mitral annular velocity) is an established diastolic function index that is not affected by hemodynamic load, but increases in 
TABLE 1: Comparison of systolic and diastolic heart failure.

\begin{tabular}{lccc}
\hline & Systolic heart failure & Diastolic heart failure \\
\hline LV remodeling & $\uparrow$ & $\begin{array}{c}\text { Concentric remodeling } \\
\text { Concentric hypertrophy }\end{array}$ \\
LV end-diastolic volume & $\uparrow$ & $\rightarrow$ \\
LV end-diastolic pressure & $\downarrow$ & $\uparrow$ \\
LV ejection fraction & $\downarrow$ & $\rightarrow$ \\
LV dP/dt & $\rightarrow$ & $\uparrow$ \\
LV stiffness & $\uparrow$ & $\uparrow$ \\
E/E' & + & $\uparrow$ \\
LA dilatation & $\uparrow$ & $\uparrow$ \\
BNP or NT-proBNP & + & + \\
\hline
\end{tabular}

LV: left ventricular; $E / E^{\prime}$ : early mitral flow velocity/early diastolic mitral annular velocity ratio; LA: left arterial; BNP: brain natriuretic peptide.

both diastolic and systolic heart failure. Pattern of pulmonary vein flow and $\mathrm{E} / \mathrm{A}$ (ratio of early to late mitral inflow peak velocity) are also established as major diastolic functional indices, though they are rather dependent on hemodynamic status [14].

In recent years, several reports on important association of various biomarkers for heart failure and subtypes of heart failure have been published [15-19]. According to those reports, plasma B-type natriuretic peptide (BNP) concentration in patients with HFpEF is lower than that in patients with HFrEF [15]. Nonetheless, at a given level of $\mathrm{BNP}$, the prognosis in patients with $\mathrm{HFpEF}$ is as poor as in those with HFrEF [15]. Not only BNP and N-terminal proBNP (NT-proBNP) but also high sensitive troponin $\mathrm{T}$ (hsTnT) was significantly associated with the risk for HFrEF [16]. On the other hand, growth differentiation factor 15 (GDF15), cystatin C, and urinary albumin excretion were significantly associated with the risk for $\operatorname{HFpEF}[16,17]$. Researchers suggest that biomarkers relevant to myocardial injury (TnT) and myocardial stress (BNP, NT-proBNP, and midregional proadrenomedullin) have significant relation with HFrEF [17-19] and biomarkers relevant to extracellular matrix remodeling (Galectin-3 and GDF15) have significant relation with HFpEF [17-19].

\section{The Pathogenesis of Diastolic Heart Failure}

The histological features of systolic heart failure include myocardial hypertrophy, loss of myocardial cells, and restructuring of the extracellular matrix. Meanwhile, significant myocardial fibrosis together with myocardial hypertrophy is typical in diastolic heart failure. Myocardial fibrosis is thought to be the main factor in increased stiffness [14].

Mechanical stimulation to the myocardium is the main factor of myocardial hypertrophy, while myocardial fibrosis may be caused by humoral factors such as various cytokines, growth factors, and hormones. In hypertensive HFpEF model rats, oxidative stress was increased and angiotensin II was produced within the arterial walls due to high blood pressure. This resulted in fibroblast activation and increased production of transforming growth factor- $\beta$ via macrophage infiltration and activation mediated by monocyte chemotactic protein-1. The resulting perivascular inflammation is reported to be the cause of reactive fibrosis of myocardium [14-16]. In Dahl salt-sensitive rat HFpEF models, it was found that endothelin, together with angiotensin II, is an important mediator of myocardial fibrosis [17].

In addition to the quantitative increase in collagen and distribution abnormalities, qualitative changes are also involved in increased myocardial stiffness caused by fibrosis. In Dahl salt-sensitive rat HFpEF models, the increase in the ratio of stiff type I collagen to type III collagen, which is highly distensible, and increased collagen cross-linking are reported to important factors of increased myocardial stiffness [18].

In diastolic heart failure, myocardial stiffness of cardiomyocytes per se also increases. Detailed mechanism for this remains unclear but is thought to be due to changes in structural proteins associated with myocardial hypertrophy. Titin, which is a giant sarcomeric protein, acts as a molecular spring and plays a large part in the distensibility of cardiomyocytes during diastole. However, in diastolic heart failure, compared with systolic heart failure, the ratio of large, distensible $\mathrm{N} 2 \mathrm{~A}$ isoforms small, rigid N2B isoforms to was found to decrease [19].

\section{Therapeutic Options}

To date, angiotensin converting enzyme inhibitors (ACEIs), angiotensin receptor blockers (ARB), beta-blockers, and statins have been tried for HFpEF. Although they are authorized for optimal treatment for HFrEF, none of them can provide the optimal treatment for HFpEF [2]. ACEI perindopril was tested for $\mathrm{HFpEF}$ patients for the first time in PEP-CHF trial [20]. It showed no difference in mortality and/or hospitalization rate for heart failure. CHARMpreserved trial, in which ARB candesartan was tested for 
cardiovascular mortality and heart failure hospitalizations, failed to demonstrate a beneficial effect on cardiovascular death but observed fewer heart failure hospitalizations in the candesartan group [21]. I-PRESERVE was so far the largest trial for HFpEF using ARB irbesartan or placebo. Mortality or hospitalization rate for cardiovascular causes was again not improved by irbesartan [22]. In OPTIMIZEHF registry, discharge use of beta-blockers exerted no effect on one-year mortality or hospitalization rate in HFpEF patients [23]. A preliminary report suggested statin therapy to be beneficial in $\mathrm{HFpEF}$ with lower mortality rate [24].

A neutral outcome in HFpEF compared with a positive outcome in HFrEF, as occurred with ACEIs, ARBs, and betablockers, might be compatible with flawed study design. However, a positive outcome in HFpEF compared with a neutral outcome in HFrEF, as occurred with statins, can no longer be attributable to study design but supports different signal transductions driving myocardial remodeling in HFpEF and HFrEF [25].

Several compounds seem to be promising for drug target of HFpEF. Phosphodiesterase 5 inhibitors (PDE5I) increase cGMP level, attenuate adrenergic stimulation, reduce ventricular-arterial stiffening, antagonize maladaptive chamber remodeling, improve endothelial function, and reduce pulmonary vascular resistance [26-30]. The PDE5I sildenafil is currently being tested in the RELAX trial, which evaluates the effects of PDE5I on exercise capacity, functional status, and ventricular function [31]. A preliminary openlabel trial documented improvements in exercise capacity and the $\mathrm{E} / \mathrm{E}^{\prime}$ ratio in $\mathrm{HFpEF}$ treated with spironolactone [32]. Aldosterone antagonists are currently being actively investigated for HFpEF in the clinical situation. Chamber stiffness is altered by the extracellular matrix like collagen. Alagebrium chloride (ALT-711) is a novel agent that breaks glucose cross-links and improves ventricular and vascular compliance in animal experiments and reduces blood pressure and vascular stiffness in humans $[33,34]$. Small open-label trial revealed that ALT-711 was associated with reduced LV mass and improved diastolic filling [35]. Diastolic dysfunction in HFpEF may be related to abnormalities in energy availability or utilization in myocytes [36-38]. Recently, abnormal ATP phosphocreatine shuttle kinetics in HFpEF was demonstrated, and similar results were recently also reported [37,39]. Currently, a novel therapy targeting energy utilization is under investigation [40].

\section{Conclusion}

Despite the fact that diastolic heart failure is a common condition of heart failure, its precise definition still remains unclear. Therefore, the similarities and differences between the pathogenesis and pathophysiology of diastolic and systolic heart failure have not been sufficiently elucidated. This is thought to be the most significant reason for a lack of established treatment methods for diastolic heart failure. We hope to proceed with future studies on this paper.

\section{Conflict of Interests}

The author declares that there is no conflict of interests regarding the publication of this article.

\section{References}

[1] M. Ouzounian, D. S. Lee, and P. P. Liu, "Diastolic heart failure: mechanisms and controversies," Nature Clinical Practice Cardiovascular Medicine, vol. 5, pp. 375-386, 2008.

[2] M. T. Maeder and D. M. Kaye, "Heart failure with normal left ventricular ejection fraction," Journal of the American College of Cardiology, vol. 53, no. 11, pp. 905-918, 2009.

[3] B. A. Borlaug and W. J. Paulus, "Heart failure with preserved ejection fraction: pathophysiology, diagnosis, and treatment," European Heart Journal, vol. 32, no. 6, pp. 670-679, 2011.

[4] E. H. García, E. R. Perna, E. F. Farías et al., "Reduced systolic performance by tissue Doppler in patients with preserved and abnormal ejection fraction: new insights in chronic heart failure," International Journal of Cardiology, vol. 108, no. 2, pp. 181-188, 2006.

[5] G. Yip, M. Wang, Y. Zhang, J. W. H. Fung, P. Y. Ho, and J. E. Sanderson, "Left ventricular long axis function in diastolic heart failure is reduced in both diastole and systole: time for a redefinition?” Heart, vol. 87, no. 2, pp. 121-125, 2002.

[6] S. J. Skaluba and S. E. Litwin, "Mechanisms of exercise intolerance: insights from tissue Doppler imaging," Circulation, vol. 109, no. 8, pp. 972-977, 2004.

[7] K. Yamamoto, Y. Sakata, T. Ohtani, Y. Takeda, and T. Mano, "Heart failure with preserved ejection fraction: what is known and unknown," Circulation Journal, vol. 73, no. 3, pp. 404-410, 2009.

[8] S. A. Hunt, W. T. Abraham, M. H. Chin et al., "ACC/AHA 2005 guideline update for the diagnosis and management of chronic heart failure in the adult: a report of the American College of Cardiology/American Heart Association Task Force on Practice Guidelines (Writing Committee to Update the 2001 Guidelines for the Evaluation and Management of Heart Failure): developed in collaboration with the American College of Chest Physicians and the International Society for Heart and Lung Transplantation: endorsed by the Heart Rhythm Society," Circulation, vol. 112, no. 12, pp. e154-e235, 2005.

[9] R. S. Vasan and D. Levy, "Defining diastolic heart failure: a call for standardized diagnostic criteria," Circulation, vol. 101, no. 17, pp. 2118-2121, 2000.

[10] M. R. Zile, W. H. Gaasch, J. D. Carroll et al., "Heart failure with a normal ejection fraction: is measurement of diastolic function necessary to make the diagnosis of diastolic heart failure?" Circulation, vol. 104, no. 7, pp. 779-782, 2001.

[11] M. R. Zile, C. F. Baicu, and W. H. Gaasch, "Diastolic heart failure-abnormalities in active relaxation and passive stiffness of the left ventricle," The New England Journal of Medicine, vol. 350, no. 19, pp. 1953-1959, 2004.

[12] T. E. Owan, D. O. Hodge, R. M. Herges, S. J. Jacobsen, V. L. Roger, and M. M. Redfield, "Trends in prevalence and outcome of heart failure with preserved ejection fraction," The New England Journal of Medicine, vol. 355, no. 3, pp. 251-259, 2006.

[13] R. S. Bhatia, J. V. Tu, D. S. Lee et al., "Outcome of heart failure with preserved ejection fraction in a population-based study," The New England Journal of Medicine, vol. 355, no. 3, pp. 260269, 2006. 
[14] S. F. Nagueh, C. P. Appleton, T. C. Gillebert et al., "Recommendations for the evaluation of left ventricular diastolic function by echocardiography," European Journal of Echocardiography, vol. 10, no. 2, pp. 165-193, 2009.

[15] D. J. van Veldhuisen, G. C. Linssen, T. Jaarsma et al., "Btype natriuretic Peptide and prognosis in heart failure patients with preserved and reduced ejection fraction," Journal of the American College of Cardiology, vol. 61, no. 14, pp. 1498-1506, 2013.

[16] F. P. Brouwers, R. A. de Boer, P. van der Harst et al., "Incidence and epidemiology of new onset heart failure with preserved vs. reduced ejection fraction in a community-based cohort: 11-year follow-up of PREVEND," European Heart Journal, vol. 34, pp. 1424-1431, 2013.

[17] R. Santhanakrishnan, J. P. Chong, T. P. Ng et al., "Growth differentiation factor 15, ST2, high-sensitivity troponin T, and $\mathrm{N}$-terminal pro brain natriuretic peptide in heart failure with preserved vs. reduced ejection fraction," European Journal of Heart Failure, vol. 14, pp. 1338-1347, 2012.

[18] R. R. J. van Kimmenade and J. L. Januzzi Jr., "Emerging biomarkers in heart failure," Clinical Chemistry, vol. 58, no. 1, pp. 127-138, 2012.

[19] H. K. Gaggin and J. L. Januzzi Jr., "Biomarkers and diagnostics in heart failure," Biochimica et Biophysica Acta, 2013.

[20] J. G. F. Cleland, M. Tendera, J. Adamus, N. Freemantle, L. Polonski, and J. Taylor, "The perindopril in elderly people with chronic heart failure (PEP-CHF) study," European Heart Journal, vol. 27, no. 19, pp. 2338-2345, 2006.

[21] S. Yusuf, M. A. Pfeffer, K. Swedberg et al., "Effects of candesartan in patients with chronic heart failure and preserved leftventricular ejection fraction: the CHARM-preserved trial," The Lancet, vol. 362, no. 9386, pp. 777-781, 2003.

[22] B. M. Massie, P. E. Carson, J. J. McMurray et al., "Irbesartan in patients with heart failure and preserved ejection fraction," The New England Journal of Medicine, vol. 359, no. 23, pp. 24562467, 2008.

[23] A. F. Hernandez, B. G. Hammill, C. M. O’Connor, K. A. Schulman, L. H. Curtis, and G. C. Fonarow, "Clinical effectiveness of beta-blockers in heart failure. Findings from the OPTIMIZEHF (Organized Program to Initiate Lifesaving Treatment in Hospitalized Patients With Heart Failure) Registry," Journal of the American College of Cardiology, vol. 53, no. 2, pp. 184-192, 2009.

[24] H. Fukuta, D. C. Sane, S. Brucks, and W. C. Little, "Statin therapy may be associated with lower mortality in patients with diastolic heart failure: a preliminary report," Circulation, vol. 112, no. 3 , pp. 357-363, 2005.

[25] W. J. Paulus and J. J. M. van Ballegoij, "Treatment of heart failure with normal ejection fraction: an inconvenient truth!," Journal of the American College of Cardiology, vol. 55, no. 6, pp. 526-537, 2010.

[26] B. A. Borlaug, V. Melenovsky, T. Marhin, P. Fitzgerald, and D. A. Kass, "Sildenafil inhibits $\beta$-adrenergic-stimulated cardiac contractility in humans," Circulation, vol. 112, no. 17, pp. 26422649, 2005.

[27] C. Vlachopoulos, K. Hirata, and M. F. O’Rourke, "Effect of sildenafil on arterial stiffness and wave reflection," Vascular Medicine, vol. 8, no. 4, pp. 243-248, 2003.

[28] S. D. Katz, K. Balidemaj, S. Homma, H. Wu, J. Wang, and S. Maybaum, "Acute type 5 phosphodiesterase inhibition with sildenafil enhances flow-mediated vasodilation in patients with chronic heart failure," Journal of the American College of Cardiology, vol. 36, no. 3, pp. 845-851, 2000.

[29] G. D. Lewis, J. Lachmann, J. Camuso et al., "Sildenafil improves exercise hemodynamics and oxygen uptake in patients with systolic heart failure," Circulation, vol. 115, no. 1, pp. 59-66, 2007.

[30] H. H. Chen, "Heart failure: a state of brain natriuretic peptide deficiency or resistance or both!," Journal of the American College of Cardiology, vol. 49, no. 10, pp. 1089-1091, 2007.

[31] M. M. Redfield, K. L. Lee, and E. Braunwald, "Evaluating the effectiveness of Sildenafil at improving health outcomes and exercise ability in people with diastolic heart failure (The RELAX Study)," NCT00763867, 2008, http://clinicaltrials.gov/.

[32] K. R. Daniel, G. Wells, K. Stewart, B. Moore, and D. W. Kitzman, "Effect of aldosterone antagonism on exercise tolerance, doppler diastolic function, and quality of life in older women with diastolic heart failure," Congestive Heart Failure, vol. 15, no. 2, pp. 68-74, 2009.

[33] P. V. Vaitkevicius, M. Lane, H. Spurgeon et al., "A crosslink breaker has sustained effects on arterial and ventricular properties in older rhesus monkeys," Proceedings of the National Academy of Sciences of the United States of America, vol. 98, no. 3, pp. 1171-1175, 2001.

[34] D. A. Kass, E. P. Shapiro, M. Kawaguchi et al., "Improved arterial compliance by a novel advanced glycation end-product crosslink breaker," Circulation, vol. 104, no. 13, pp. 1464-1470, 2001.

[35] W. C. Little, M. R. Zile, D. W. Kitzman, W. G. Hundley, T. X. O'Brien, and R. C. Degroof, "The effect of alagebrium chloride (ALT-711), a novel glucose cross-link breaker, in the treatment of elderly patients with diastolic heart failure," Journal of Cardiac Failure, vol. 11, no. 3, pp. 191-195, 2005.

[36] B. A. Borlaug, V. Melenovsky, S. D. Russell et al., "Impaired chronotropic and vasodilator reserves limit exercise capacity in patients with heart failure and a preserved ejection fraction," Circulation, vol. 114, no. 20, pp. 2138-2147, 2006.

[37] T. T. Phan, K. Abozguia, G. Nallur Shivu et al., "Heart failure with preserved ejection fraction is characterized by dynamic impairment of active relaxation and contraction of the left ventricle on exercise and associated with myocardial energy deficiency," Journal of the American College of Cardiology, vol. 54, no. 5, pp. 402-409, 2009.

[38] D. W. Kitzman, M. B. Higginbotham, F. R. Cobb, K. H. Sheikh, and M. J. Sullivan, "Exercise intolerance in patients with heart failure and preserved left ventricular systolic function: failure of the Frank-Starling mechanism," Journal of the American College of Cardiology, vol. 17, no. 5, pp. 1065-1072, 1991.

[39] C. S. Smith, P. A. Bottomley, S. P. Schulman, G. Gerstenblith, and R. G. Weiss, "Altered creatine kinase adenosine triphosphate kinetics in failing hypertrophied human myocardium," Circulation, vol. 114, no. 11, pp. 1151-1158, 2006.

[40] M. Frenneaux, "Perhexiline therapy in heart failure with preserved ejection fraction syndrome," NCT00839228, 2009, http://clinicaltrials.gov/. 


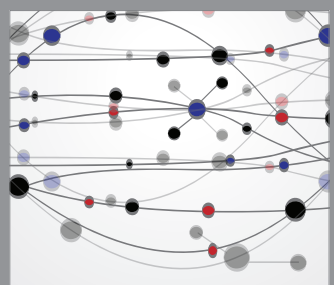

The Scientific World Journal
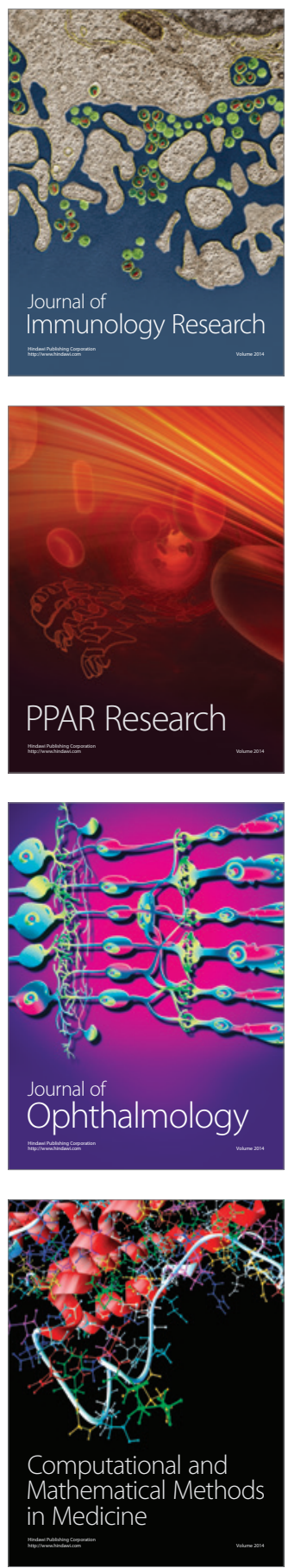

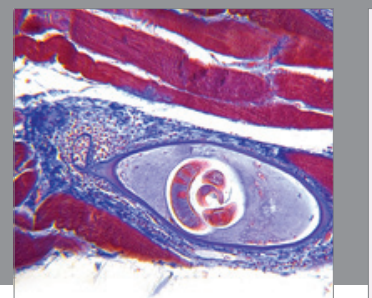

Gastroenterology

Research and Practice
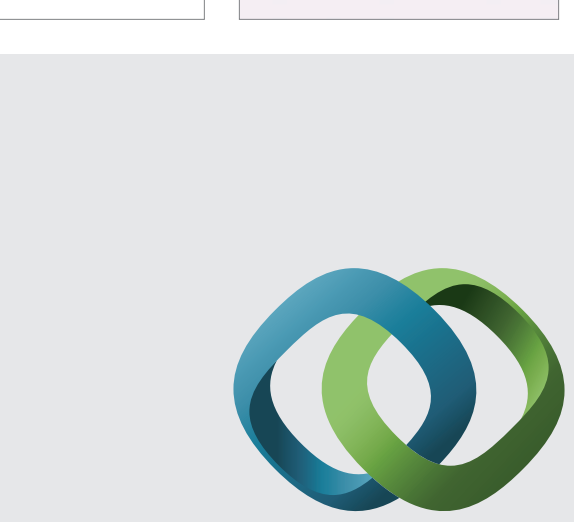

\section{Hindawi}

Submit your manuscripts at

http://www.hindawi.com
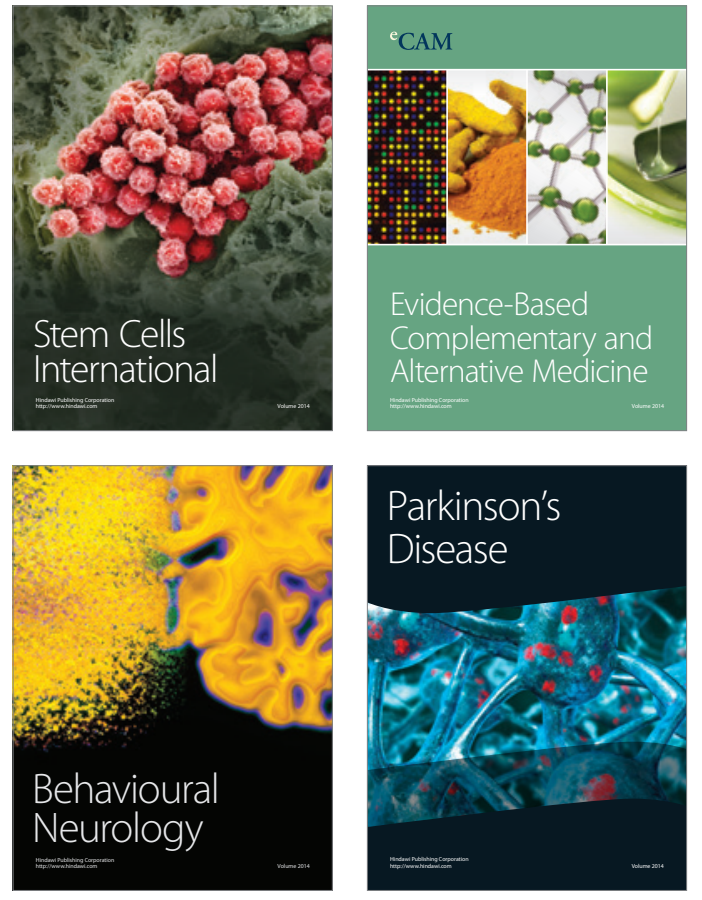
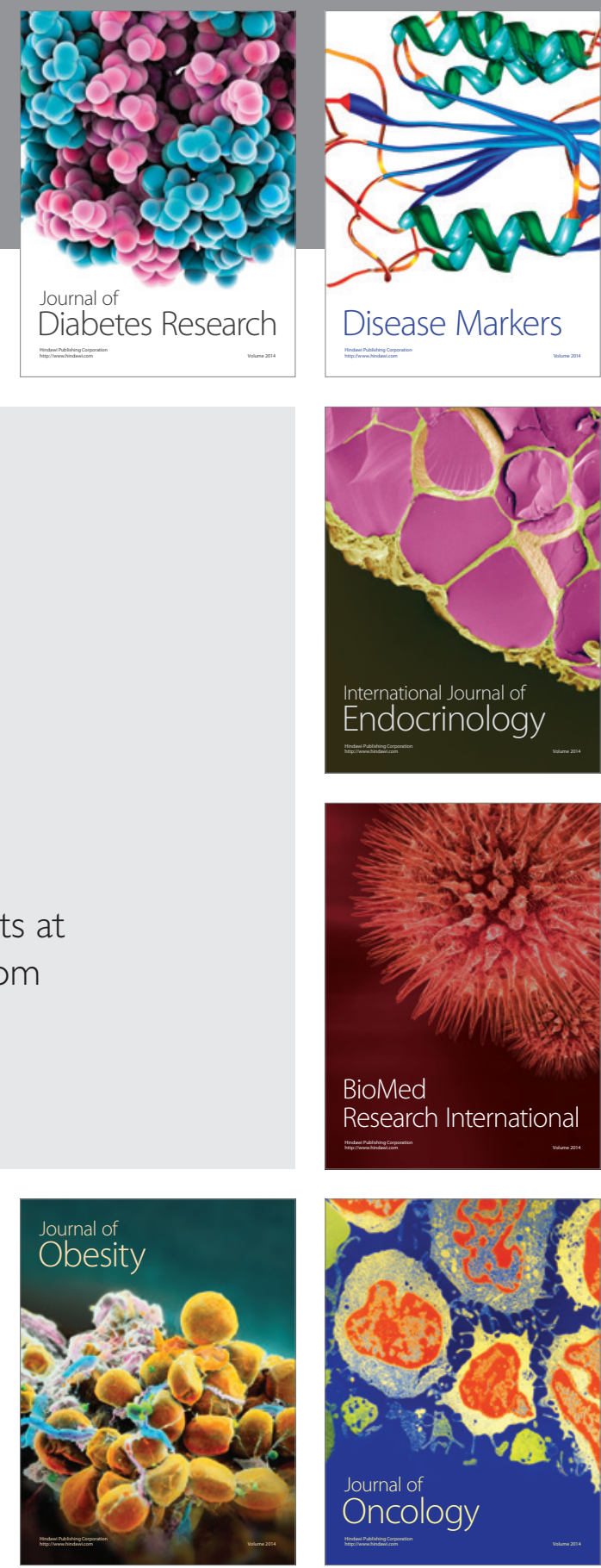

Disease Markers
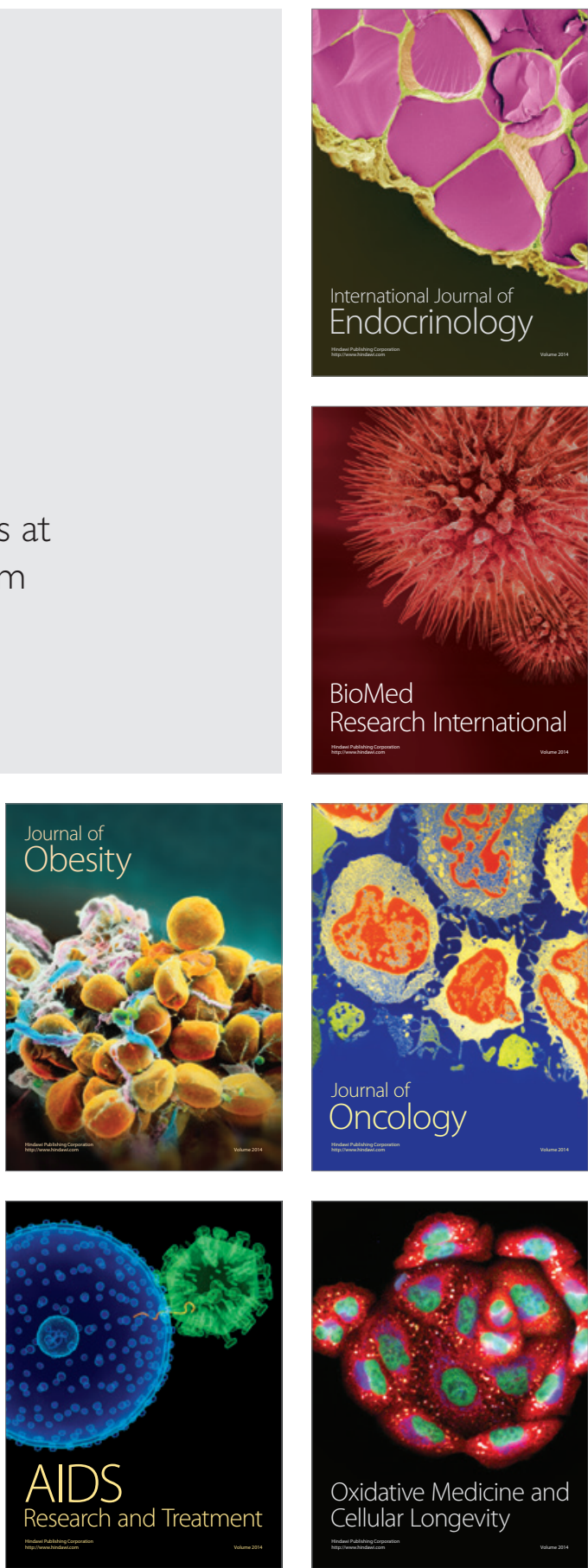